ANNALES

POLONICI MATHEMATICI

$87(2005)$

\title{
Zeta functions and blow-Nash equivalence
}

\author{
by Goulwen Fichou (Rennes)
}

\begin{abstract}
We propose a refinement of the notion of blow-Nash equivalence between Nash function germs, which has been introduced in [2] as an analog in the Nash setting of the blow-analytic equivalence defined by T.-C. Kuo [13]. The new definition is more natural and geometric. Moreover, this equivalence relation still does not admit moduli for a Nash family of isolated singularities. But though the zeta functions constructed in [2] are no longer invariants for this new relation, thanks to a Denef \& Loeser formula coming from motivic integration in a Nash setting, we manage to derive new invariants for this equivalence relation.
\end{abstract}

The classification of real analytic function germs is a difficult topic, especially as regards the choice of a good equivalence relation between germs. Even in the particular case when the analytic function germs are Nash, that is, they are moreover semi-algebraic, the difficulty still remains.

In [2], we have defined blow-Nash equivalence between Nash function germs, as an approximation with algebraic data of the blow-analytic equivalence defined by T.-C. Kuo in [13]. The latter has already been studied with slightly different definitions (see in particular S. Koike \& A. Parusiński [11] and T. Fukui \& L. Paunescu [7]). Roughly speaking, two given real analytic function germs are blow-analytic equivalent if they are topologicaly equivalent and moreover, after suitable modifications, they become analytically equivalent.

For the case of Nash function germs, the definition of blow-Nash equivalence runs as follows. Let $f, g:\left(\mathbb{R}^{d}, 0\right) \rightarrow(\mathbb{R}, 0)$ be Nash function germs. They are said to be blow-Nash equivalent if there exist two Nash modifications (we refer to Definition 1.1 for the precise definition)

$$
\pi_{f}:\left(M_{f}, \pi_{f}^{-1}(0)\right) \rightarrow\left(\mathbb{R}^{d}, 0\right) \quad \text { and } \pi_{g}:\left(M_{g}, \pi_{g}^{-1}(0)\right) \rightarrow\left(\mathbb{R}^{d}, 0\right),
$$

and a Nash isomorphism $\phi:\left(M_{f}, \pi_{f}^{-1}(0)\right) \rightarrow\left(M_{g}, \pi_{g}^{-1}(0)\right)$, that is, $\phi$ is a real analytic isomorphism with semi-algebraic graph, which induces

2000 Mathematics Subject Classification: 14B05, 14P20, 14P25, 32S15.

Key words and phrases: blow-Nash equivalence, zeta function, arc-symmetric set. 
a homeomorphism $h$ between neighbourhoods of 0 in $\mathbb{R}^{d}$ such that $f=$ $g \circ h$.

For a stronger notion of blow-Nash equivalence, we know computable invariants, which seem to be efficient tools to distinguish blow-Nash type [2, 3]. These invariants, called zeta functions (cf. Section 2.2), are constructed in a similar way to the motivic zeta functions of Denef \& Loeser, using the virtual Poincaré polynomial of arc-symmetric sets as a generalized Euler characteristic (cf. Section 2.1).

However, the definition of blow-Nash equivalence given in [2] is strong and technical. In particular the modifications are required to be algebraic, which is not natural in the Nash setting. The weaker definition of blow-Nash equivalence introduced in this paper is more natural and geometric. It is closer to the definition of blow-analytic equivalence considered by S. Koike and A. Parusiński in [11]. Moreover, it is an equivalence relation (Proposition 1.3). It is a crucial fact to prove that this equivalence relation has a good behaviour with respect to families of Nash function germs. In this direction, Theorem 1.5 states that a family with isolated singularities does not admit moduli. This result is more general that the one in [2], but the proof is just a refinement of the former one. We also mention in Section 1.2 various criteria to ensure the blow-Nash triviality of a given family.

Recently, invariants for this kind of equivalence relation have been introduced (see [6] for a survey). In particular, we defined in [2] zeta functions, following ideas coming from motivic integration [1], via the virtual Poincaré polynomial [15].

Unfortunately, although the definition of blow-Nash equivalence in this paper is more natural and geometric, the zeta functions are no longer invariants in general. However, one can derive new invariants from these zeta functions by evaluating their coefficients, which are rational functions in the indeterminate $u$ with coefficients in $\mathbb{Z}$, at convenient values (cf. Theorem 3.4). As a key ingredient, we generalize the Denef \& Loeser formulae, which express the zeta functions in terms of a modification, to the setting of Nash modifications (see Section 2.3).

As an application, we manage to distinguish the blow-Nash type of some Brieskorn polynomials whose blow-analytic type is not even known!

Acknowledgements. The author wishes to thank T. Fukui, S. Koike and A. Parusiński for valuable discussions on the subject.

\section{Blow-Nash equivalence}

1.1. Let us begin by stating the definition of blow-Nash equivalence between Nash function germs that we consider in this paper. It is a natural 
adaptation of the blow-analytic equivalence defined by T.-C. Kuo ([13]) to the Nash framework.

\section{DEFINITION 1.1.}

(1) Let $f:\left(\mathbb{R}^{d}, 0\right) \rightarrow(\mathbb{R}, 0)$ be a Nash function germ. A Nash modification of $f$ is a proper surjective Nash map $\pi:\left(M, \pi^{-1}(0)\right) \rightarrow\left(\mathbb{R}^{d}, 0\right)$ whose complexification $\pi^{*}$ is an isomorphism everywhere except on some thin subset of $M^{*}$, and such that $f \circ \pi$ has only normal crossings.

(2) Two given germs of Nash functions $f, g:\left(\mathbb{R}^{d}, 0\right) \rightarrow(\mathbb{R}, 0)$ are said to be blow-Nash equivalent if there exist two Nash modifications

$$
\sigma_{f}:\left(M_{f}, \sigma_{f}^{-1}(0)\right) \rightarrow\left(\mathbb{R}^{d}, 0\right) \text { and } \sigma_{g}:\left(M_{g}, \sigma_{g}^{-1}(0)\right) \rightarrow\left(\mathbb{R}^{d}, 0\right),
$$

and a Nash isomorphism $\Phi$ between semi-algebraic neighbourhoods $\left(M_{f}, \sigma_{f}^{-1}(0)\right)$ and $\left(M_{g}, \sigma_{g}^{-1}(0)\right)$ which induces a homeomorphism $\phi$ : $\left(\mathbb{R}^{d}, 0\right) \rightarrow\left(\mathbb{R}^{d}, 0\right)$ such that the diagram

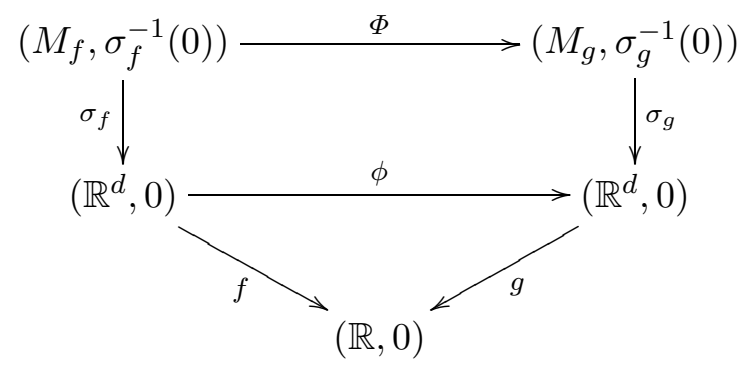

is commutative.

REMARK 1.2 .

(1) Let us specify some classical terminology (see [6] for example). A homeomorphism $\phi$ as above is called a blow-Nash homeomorphism. If, as in [2], we ask moreover $\Phi$ to preserve the multiplicities of the jacobian determinant along the exceptional divisors of the Nash modifications $\sigma_{f}, \sigma_{g}$, then $\Phi$ is called a blow-Nash isomorphism.

Note that there exist blow-Nash homeomorphisms which are not blow-Nash isomorphisms (see [6]).

(2) In [2], we consider a more specjal notion of blow-Nash equivalence. Namely, the Nash modifications are replaced by proper algebraic birational morphisms and the blow-Nash homeomorphism is moreover required to be a blow-Nash isomorphism. Definition 1.1 is more natural since all the data are of Nash class.

The proof of the following result is a direct analog of the corresponding one in [13]. 
Proposition 1.3. Blow-Nash equivalence is an equivalence relation between Nash function germs.

Proof. The point is the transitivity property. Let $f_{1}, f_{2}, f_{3}:\left(\mathbb{R}^{d}, 0\right) \rightarrow$ $(\mathbb{R}, 0)$ be Nash function germs such that $f_{1} \sim f_{2}$ and $f_{2} \sim f_{3}$. Let $\sigma_{1}, \sigma_{2}$ and $\sigma_{2}^{\prime}, \sigma_{3}^{\prime}$ be Nash modifications, and $\phi, \phi^{\prime}$ be homeomorphisms as in Definition 1.1 for $f_{1}, f_{2}$ and $f_{2}, f_{3}$ respectively. The fiber product $M$ (respectively $M^{\prime}$ ) of $\phi \circ \sigma_{1}$ and $\sigma_{2}$ (respectively $\phi^{\prime} \circ \sigma_{2}^{\prime}$ and $\sigma_{3}^{\prime}$ ) gives suitable Nash modifications of $\left(\mathbb{R}^{d}, 0\right)$. Taking once more the fiber product $M^{\prime \prime}$ of $M$ and $M^{\prime}$ solves the problem since the compositions of the projections with the initial modifications $\sigma_{1}$ and $\sigma_{3}^{\prime}$ remain Nash modifications for $f_{1}$ and $f_{3}$.

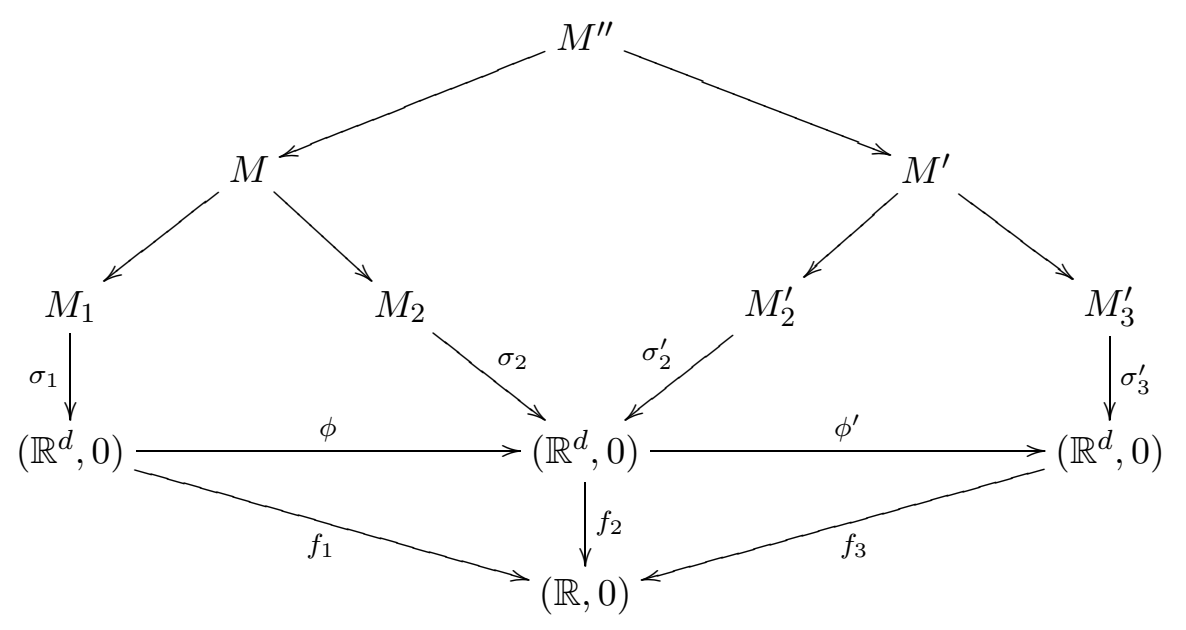

REMARK 1.4. Note that for the blow-Nash equivalence considered in [2], we had to consider the equivalence relation generated by a similar relation. This difficulty came from the fact that the fiber product of an algebraic map and a Nash map need not be algebraic. The point here is that the fiber product of Nash maps still remains in the Nash class.

The question of moduli is a natural and crucial issue when one studies an equivalence relation between germs. The following theorem states that blow-Nash equivalence has a good behaviour with respect to families of Nash function germs. More precisely, blow-Nash equivalence does not admit moduli for a Nash family of Nash function germs with an isolated singularity. Denote by $P$ the cube $[0,1]^{k}$ for an integer $k$.

Theorem 1.5. Let $F:\left(\mathbb{R}^{d}, 0\right) \times P \rightarrow(\mathbb{R}, 0)$ be a Nash map and assume that $F(\cdot, p):\left(\mathbb{R}^{d}, 0\right) \rightarrow \mathbb{R}$ has an isolated singularity at 0 for each $p \in P$. Then the family $F(\cdot, p)$, for $p \in P$, consists of a finite number of blow-Nash equivalence classes. 
REMARK 1.6. The proof of Theorem 1.5 can be performed in a similar way to the one in [2], even if the result is more general here. Indeed, in [2] we had to restrict the study to particular Nash families, namely families which admit, as a resolution of the singularities, an algebraic modification. But if we allow the modifications to become Nash, Hironaka's resolution of singularities provides us suitable Nash modifications [9].

1.2. Blow-Nash triviality. In view of classification problems, a worthwhile issue is to give criteria for a Nash family to consist of a unique blowNash class. In particular, one says that a Nash family $F:\left(\mathbb{R}^{d}, 0\right) \times P \rightarrow(\mathbb{R}, 0)$ is blow-Nash trivial if there exist a Nash modification $\sigma:(M, E) \rightarrow\left(\mathbb{R}^{d}, 0\right)$, a $t$-level preserving homeomorphism $\varphi:\left(\mathbb{R}^{d}, 0\right) \times P \rightarrow\left(\mathbb{R}^{d}, 0\right) \times P$ and a $t$-level preserving Nash isomorphism $\Phi:(M, E) \times P \rightarrow(M, E) \times P$ such that the diagram

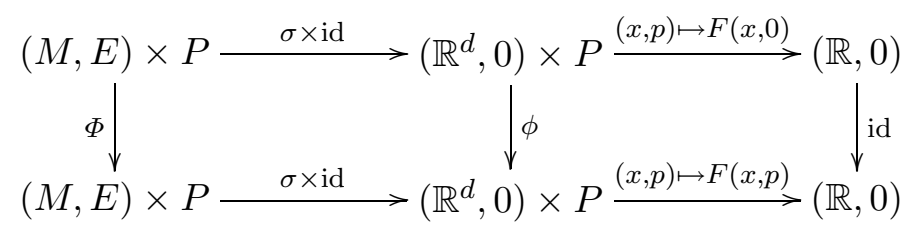

is commutative.

Below, we mention sufficient conditions to ensure the blow-Nash triviality of a given family, which are analogs of corresponding results concerning blow-analytic equivalence ([7], [8]). Moreover their proof (cf. Remark 1.10) is a direct consequence of the one of Theorem 1.5.

Let us introduce some terminology before stating the first result, which is inspired by the main theorem of [8]. For an analytic function germ $f$ : $\left(\mathbb{R}^{d}, 0\right) \rightarrow(\mathbb{R}, 0)$, denote by $\sum_{I} c_{I} x^{I}$ its Taylor expansion at the origin, where $x^{I}=x_{1}^{i_{1}} \cdots x_{d}^{i_{d}}, I=\left(i_{1}, \ldots, i_{d}\right)$. The Newton polygon of $f$ is the convex hull of the union of the sets $I+\mathbb{R}_{+}^{d}$ for those $|I|$ such that $c_{I} \neq 0$. For a face $\gamma$ of this polyhedron, we put $f_{\gamma}(x)=\sum_{I \in \gamma} c_{I} x^{I}$. The germ $f$ is said to be nondegenerate, with respect to its Newton polygon, if the only singularities of $f_{\gamma}$ are concentrated in the coordinate hyperplanes, for any compact face $\gamma$ of the Newton polygon. Finally, one says that a given face is a coordinate face if it is parallel to some coordinate hyperplane.

Proposition 1.7. Assume that the Newton polygon of $F(\cdot, p)$ is independent of $p \in P$, nondegenerate for each $p \in P$, and that $(F(\cdot, p))_{\gamma}$ is independent of $p \in P$ for any noncompact and noncoordinate face $\gamma$ of the Newton polygon. Then the family $\{F(\cdot, p)\}_{p \in P}$ is blow-Nash trivial.

The second result is inspired by the main theorem in [7]. Consider the Taylor expansion $F(x, p)=\sum_{I} c_{I}(p) x^{I}$ of $F$ at the origin of $\mathbb{R}^{d}$. For a $d$-uple 
of positive integers $w=\left(w_{1}, \ldots, w_{d}\right)$, we set

$$
H_{i}^{(w)}(x, p)=\sum_{I:|I|_{w}=i} c_{I} x^{I}, \quad \text { where } \quad|I|_{w}=i_{1} w_{1}+\cdots+i_{d} w_{d} .
$$

Denote by $m$ the smallest integer $i$ such that $H_{i}^{(w)}(x, p)$ is not identically equal to 0 .

Proposition 1.8. If there exists a d-uple of positive integers $w$ such that $H_{m}^{(w)}(x, p)$ has an isolated singularity at the origin of $\mathbb{R}^{d}$ for any $p \in P$, then the family $\{F(\cdot, p)\}_{p \in P}$ is blow-Nash trivial.

EXAmple $1.9([7])$. Let $F:\left(\mathbb{R}^{3}, 0\right) \times \mathbb{R} \rightarrow(\mathbb{R}, 0)$ be the Briançon-Speder family, that is,

$$
F(x, y, z, p)=z^{5}+p y^{6} z+x y^{7}+x^{15} .
$$

This family is weighted homogeneous with weight $(1,2,3)$ and weighted degree 15. Moreover it defines an isolated singularity at the origin for $p \neq$ $p_{0}=-15^{1 / 7}(7 / 2)^{4 / 5} / 3$. Therefore the Briançon-Speder family is blow-Nash trivial over each interval that does not contain $p_{0}$.

REMARK 1.10. The proof of these triviality results in the blow-analytic case is based on integration along an analytic vector field defined on the parameter space, and which can be lifted through the modification. The flow of the lifted vector field gives the trivialization upstairs. Moreover the assumptions made enable one to choose, as a modification, a toric modification that has a unique critical value at the origin of $\mathbb{R}^{d}$. Therefore the trivialization upstairs induces a trivialization at the level of the parameter space.

However, by integration along a Nash vector field, one may not keep Nash data, and therefore the same method as in the blow-analytic case does not apply in the situation of Propositions 1.7, 1.8. However, one can replace this integration by the following argument (presented in detail in [2]). First, resolve the singularities of the family via the relevant toric modification as in [7], [8]. Then trivialize the zero level of the function germs with the Nash Isotopy Lemma [5]. Finally, trivialize the $t$-levels, $t \neq 0$, via well-chosen projections that can be proven to be of blow-Nash class.

2. Zeta functions. In this section, we recall the definition of the naive zeta function of a Nash function germ (as defined in [2]). Then we prove the so-called Denef \& Loeser formula for such a zeta function in terms of a Nash modification. This result is new and requires generalizing the change of variables formula in the theory of motivic integration to the Nash setting.

2.1. Virtual Poincaré polynomial of arc-symmetric sets. Arc-symmetric sets have been introduced by K. Kurdyka [14] in 1988 in order to study "rigid 
components" of real algebraic varieties. The category of arc-symmetric sets contains the real algebraic varieties and, in some sense, this category has a better behaviour than that of real algebraic varieties, maybe closer to complex algebraic varieties. For a detailed treatment of arc-symmetric sets, we refer to [2]. Nevertheless, let us give a precise definition of such sets.

We fix a compactification of $\mathbb{R}^{n}$, for instance $\mathbb{R}^{n} \subset \mathbb{P}^{n}$.

Definition 2.1. Let $A \subset \mathbb{P}^{n}$ be a semi-algebraic set. We say that $A$ is arc-symmetric if, for every real analytic arc $\gamma:]-1,1\left[\rightarrow \mathbb{P}^{n}\right.$ such that $\gamma(]-1,0[) \subset A$, there exists $\varepsilon>0$ such that $\gamma(] 0, \varepsilon[) \subset A$.

One can think about arc-symmetric sets as the biggest category, denoted $\mathcal{A S}$, stable under boolean operations and containing the compact real algebraic varieties and their connected components.

In particular, the following lemma specifies what the nonsingular arcsymmetric sets are. Note that by an isomorphism between arc-symmetric sets, we mean a birational map containing the arc-symmetric sets in the support. Moreover, a nonsingular arc-symmetric set is an arc-symmetric set whose intersection with the singular locus of its Zariski closure is empty.

Lemma $2.2([2])$. Compact nonsingular arc-symmetric sets are isomorphic to unions of connected components of compact nonsingular real algebraic varieties.

A Nash isomorphism between arc-symmetric sets $A_{1}, A_{2} \in \mathcal{A S}$ is the restriction of an analytic and semi-algebraic isomorphism between compact semi-algebraic and real analytic sets $B_{1}, B_{2}$ containing $A_{1}, A_{2}$ respectively. Generalized Euler characteristics for arc-symmetric sets are invariants, under Nash isomorphisms, which enable one to give concrete measures in the theory of motivic integration. A generalized Euler characteristic is defined as follows.

An additive map on $\mathcal{A S}$ with values in an abelian group is a map $\chi$ defined on $\mathcal{A S}$ such that

(1) for arc-symmetric sets $A$ and $B$ which are Nash isomorphic, $\chi(A)=$ $\chi(B)$,

(2) for a closed arc-symmetric subset $B$ of $A, \chi(A)=\chi(B)+\chi(A \backslash B)$. If moreover $\chi$ takes its values in a commutative ring and satisfies $\chi(A \times B)=$ $\chi(A) \cdot \chi(B)$ for arc-symmetric sets $A, B$, then we say that $\chi$ is a generalized Euler characteristic on $\mathcal{A S}$.

In [2] we proved:

Proposition 2.3. There exist additive maps on $\mathcal{A S}$ with values in $\mathbb{Z}$, denoted $\beta_{i}$ and called virtual Betti numbers, that coincide with the classical Betti numbers $\operatorname{dim} H_{i}(\cdot, \mathbb{Z} / 2 \mathbb{Z})$ on the connected components of compact 
nonsingular real algebraic varieties. Moreover $\beta(\cdot)=\sum_{i \geq 0} \beta_{i}(\cdot) u^{i}$ is a generalized Euler characteristic on $\mathcal{A S}$, with values in $\mathbb{Z}[u]$.

EXAMPLE 2.4. If $\mathbb{P}^{k}$ denotes the real projective space of dimension $k$, which is nonsingular and compact, then $\beta\left(\mathbb{P}^{k}\right)=1+u+\cdots+u^{k}$. Now, compactify the affine line $\mathbb{A}_{\mathbb{R}}^{1}$ to $\mathbb{P}^{1}$ by adding one point at infinity. By additivity $\beta\left(\mathbb{A}_{\mathbb{R}}^{1}\right)=\beta\left(\mathbb{P}^{1}\right)-\beta$ (point) $=u$, and so $\beta\left(\mathbb{A}_{\mathbb{R}}^{k}\right)=u^{k}$.

REMARK 2.5.

(1) The virtual Poincaré polynomial is not a topological invariant (cf. [15]).

(2) The virtual Poincaré polynomial $\beta$ respects the dimension of arcsymmetric sets: for $A \in \mathcal{A S}, \operatorname{dim}(A)=\operatorname{deg}(\beta(A))$. In particular, this ensures that a nonempty arc-symmetric set has a nonzero value under the virtual Poincaré polynomial.

(3) By evaluating $u$ at -1 , one recovers the classical Euler characteristic with compact supports $([2,15])$.

2.2. Zeta functions. The zeta functions of a Nash function germ are defined by taking the value, under the virtual Poincaré polynomial, of certain sets of arcs related to the germ.

Denote by $\mathcal{L}$ the space of formal arcs at the origin $0 \in \mathbb{R}^{d}$, defined by

$$
\mathcal{L}=\mathcal{L}\left(\mathbb{R}^{d}, 0\right)=\left\{\gamma:(\mathbb{R}, 0) \rightarrow\left(\mathbb{R}^{d}, 0\right): \gamma \text { formal }\right\},
$$

and by $\mathcal{L}_{n}$, for an integer $n$, the space of $\operatorname{arcs}$ truncated at the order $n+1$ :

$$
\mathcal{L}_{n}=\left\{\gamma(t)=a_{1} t+a_{2} t^{2}+\cdots+a_{n} t^{n}: a_{i} \in \mathbb{R}^{d}\right\} .
$$

Let $\pi_{n}: \mathcal{L} \rightarrow \mathcal{L}_{n}$ be the truncation morphism.

Consider a Nash function germ $f:\left(\mathbb{R}^{d}, 0\right) \rightarrow(\mathbb{R}, 0)$. We define the naive zeta function $Z_{f}(u, T)$ of $f$ as the following element of $\mathbb{Z}\left[u, u^{-1}\right][[T]]$ :

$$
Z_{f}(u, T)=\sum_{n \geq 1} \beta\left(\mathcal{X}_{n}\right) u^{-n d} T^{n},
$$

where $\mathcal{X}_{n}$ is composed of those arcs that, composed with $f$, give a series with order $n$ :

$$
\mathcal{X}_{n}=\left\{\gamma \in \mathcal{L}_{n}: \operatorname{ord}(f \circ \gamma)=n\right\}=\left\{\gamma \in \mathcal{L}_{n}: f \circ \gamma(t)=b t^{n}+\cdots, b \neq 0\right\} .
$$

Similarly, we define zeta functions with sign by

$$
Z_{f}^{+}(u, T)=\sum_{n \geq 1} \beta\left(\mathcal{X}_{n}^{+}\right) u^{-n d} T^{n}, \quad Z_{f}^{-}(u, T)=\sum_{n \geq 1} \beta\left(\mathcal{X}_{n}^{-}\right) u^{-n d} T^{n}
$$

where

$$
\mathcal{X}_{n}^{ \pm}=\left\{\gamma \in \mathcal{L}_{n}: f \circ \gamma(t)= \pm t^{n}+\cdots\right\} .
$$

We remark that $\mathcal{X}_{n}, \mathcal{X}_{n}^{ \pm}$, for $n \geq 1$, are constructible subsets of $\mathbb{R}^{n d}$, hence belong to $\mathcal{A S}$. 
In [2], we prove that these zeta functions are invariants for the stronger notion of blow-Nash equivalence (with blow-Nash isomorphism). Adapted to the present case, what we will prove is:

Proposition 2.6. Let $f, g:\left(\mathbb{R}^{d}, 0\right) \rightarrow(\mathbb{R}, 0)$ be germs of Nash functions. If $f$ and $g$ are blow-Nash equivalent via a blow-Nash isomorphism, then

$$
Z_{f}(u, T)=Z_{g}(u, T), \quad Z_{f}^{ \pm}(u, T)=Z_{g}^{ \pm}(u, T) .
$$

REMARK 2.7.

(1) We do not know whether or not the zeta functions are invariant for blow-Nash equivalence.

(2) The above result is a step toward the resolution of the main issue of the paper (Theorem 3.4): what information is preserved, at the level of zeta functions, if a blow-Nash homeomorphism replaces a blow-Nash isomorphism.

(3) Note that if the modifications appearing in the definition of the blowNash equivalence of $f$ and $g$ are moreover algebraic, the result is precisely the one in [2]. So what we have to justify here is that Nash modifications are allowed. The key point is the Denef \& Loeser formula (cf. next section).

2.3. Denef \& Loeser formula for a Nash modification. The key ingredient of the proof of Proposition 2.6, which will also be crucial in Section 3, is the following Denef \& Loeser formula which expresses the zeta function of a Nash function germ in terms of a modification of its zero locus. First, we state the case of the naive zeta function.

Proposition 2.8 (Denef \& Loeser formula). Let $\sigma:\left(M, \sigma^{-1}(0)\right) \rightarrow$ $\left(\mathbb{R}^{d}, 0\right)$ be a Nash modification of $\mathbb{R}^{d}$ such that $f \circ \sigma$ and the jacobian determinant jac $\sigma$ have only normal crossings simultaneously, and assume moreover that $\sigma$ is an isomorphism over the complement of the zero locus of $f$. Let $(f \circ \sigma)^{-1}(0)=\bigcup_{j \in J} E_{j}$ be the decomposition of $(f \circ \sigma)^{-1}(0)$ into irreducible components, and assume that $\sigma^{-1}(0)=\bigcup_{k \in K} E_{k}$ for some $K \subset J$. Put $N_{i}=$ mult $_{E_{i}} f \circ \sigma$ and $\nu_{i}=1+\operatorname{mult}_{E_{i}}$ jac $\sigma$, and, for $I \subset J$, set

$$
E_{I}^{0}=\left(\bigcap_{i \in I} E_{i}\right) \backslash\left(\bigcup_{j \in J \backslash I} E_{j}\right) .
$$

Then

$$
Z_{f}(u, T)=\sum_{I \neq \emptyset}(u-1)^{|I|} \beta\left(E_{I}^{0} \cap \sigma^{-1}(0)\right) \Phi_{I}(T)
$$

where

$$
\Phi_{I}(T)=\prod_{i \in I} \frac{u^{-\nu_{i}} T^{N_{i}}}{1-u^{-\nu_{i}} T^{N_{i}}} .
$$


In the case with sign, let us define first coverings of the exceptional strata $E_{I}^{0}$ as follows.

Let $U$ be an affine open subset of $M$ such that $f \circ \sigma=u \prod_{i \in I} y_{i}^{N_{i}}$ on $U$, where $u$ is a Nash function that does not vanish. Put

$$
R_{U}^{ \pm}=\left\{(x, t) \in\left(E_{I}^{0} \cap U\right) \times \mathbb{R}: t^{m}= \pm 1 / u(x)\right\},
$$

where $m=\operatorname{gcd}\left(N_{i}\right)$. Then the $R_{U}^{ \pm}$glue together along the $E_{I}^{0} \cap U$ to give $\widetilde{E_{I}^{0, \pm}}$.

Proposition 2.9. With the assumptions and notation of Proposition 2.8 , one can express the zeta functions with sign in terms of a Nash modification as

$$
\left.Z_{f}^{ \pm}(T)=\sum_{I \neq \emptyset}(u-1)^{|I|-1} \beta \widetilde{\left(\widetilde{E_{I}^{0, \pm}}\right.} \cap \sigma^{-1}(0)\right) \prod_{i \in I} \frac{u^{-\nu_{i}} T^{N_{i}}}{1-u^{-\nu_{i}} T^{N_{i}}} .
$$

Remark 2.10. The proofs of Propositions 2.8 and 2.9 in the Nash case run as in the algebraic case (cf. [2] for example, which is already an adaptation to the real case of [1]). In particular, in the remainder of this section, we prove that we can apply the same method. The main point is that we have at our disposal a Kontsevich change of variables formula in the Nash case. In order to prove this, the following lemma is crucial.

Lemma 2.11. Let $h:(M, E) \rightarrow\left(\mathbb{R}^{d}, 0\right)$ be a proper surjective Nash map. Put

$$
\Delta_{e}=\left\{\gamma \in \mathcal{L}(M, E): \operatorname{ord}_{t} \mathrm{jac}(\gamma(t))=e\right\}
$$

for an integer $e \geq 1$, where $\mathcal{L}(M, E)$ is the space of formal arcs on $M$ with origin in $E$, and put $\Delta_{e, n}=\pi_{n}\left(\Delta_{e}\right)$. If $e \geq 1$ and $n \geq 2 e$, then $h_{n}\left(\Delta_{e, n}\right)$ is arc-symmetric and $h_{n}$ is a piecewise trivial fibration over $\Delta_{e, n}$, where the pieces are arc-symmetric sets, with fiber $\mathbb{R}^{e}$.

As an intermediate result, note the following elementary lemma whose proof is based on Taylor's formula (cf. [1]).

Lemma 2.12. Let $e \geq 1$ and $n \geq 2 e$. If $\gamma_{1}, \gamma_{2} \in \mathcal{L}(M, E), \gamma_{1} \in \Delta_{e}$ and $h\left(\gamma_{1}\right) \equiv h\left(\gamma_{2}\right) \bmod t^{n+1}$ then $\gamma_{2} \in \Delta_{e}$ and $\gamma_{1} \equiv \gamma_{2} \bmod t^{n-e+1}$.

Proof of Lemma 2.11. It follows from Lemma 2.12 that $h_{n}$ is injective when restriced to $\Delta_{e, n} \cap \pi_{n-e}(\mathcal{L}(M, E))$, and that $h_{n}\left(\Delta_{e, n} \cap \pi_{n-e}(\mathcal{L}(M, E))\right)$ $=h_{n}\left(\Delta_{e, n}\right)$. Then $h_{n}\left(\Delta_{e, n}\right)$ is arc-symmetric, being the image under an injective Nash map of an arc-symmetric set (more precisely, a constructible set).

Now, the remainder of the proof can be carried out exactly as in [1].

To obtain the Kontsevich change of variables formula for a Nash modification, and therefore Propositions 2.8 and 2.9, it suffices to follow the 
same computation as in [2]. Indeed, Lemma 2.11 enables us to apply word for word the method given in [2], just by replacing "constructible sets" by "arc-symmetric sets".

Now we can detail the proof of Proposition 2.6.

Proof of Proposition 2.6. Let us prove the proposition in the case of the naive zeta functions.

Let $f, g:\left(\mathbb{R}^{d}, 0\right) \rightarrow(\mathbb{R}, 0)$ be blow-Nash equivalent Nash function germs. By definition of blow-Nash equivalence, there exist two Nash modifications, joined by a commutative diagram as in Definition 1.1.

By a sequence of blowings-up with smooth Nash centres, one can make the jacobian determinants have only normal crossings. One can assume moreover that the exceptional divisors also have only normal crossings with the ones of the previous Nash modifications, so that we are in the situation where we can apply the Denef \& Loeser formula.

Then it is sufficient to prove that the expressions of the zeta functions of the germs, obtained via the Denef \& Loeser formula, coincide. Now, the terms of the form $\beta\left(E_{I}^{0} \cap \sigma^{-1}(0)\right)$ are equal since the virtual Poincaré polynomial $\beta$ is invariant under Nash isomorphisms (cf. Proposition 2.3) and the $N_{i}$ remain the same because of the commutativity of the diagram (cf. Definition 1.1). Finally, the $\nu_{i}$ coincide due to the additional assumption that the blow-Nash homeomorphism is a blow-Nash isomorphism.

3. Evaluating the zeta functions. In order to perform a classification of Nash function germs under blow-Nash equivalence, one needs invariants for this equivalence relation. The only ones known until now are the Fukui invariants [10] and the zeta functions of Koike-Parusiński defined with the Euler characteristic with compact supports [11]. However, for the stronger notion of blow-Nash equivalence, the zeta functions obtained via the virtual Poincaré polynomial are also invariants (cf. Proposition 2.6).

In this section, we define new invariants for blow-Nash equivalence. They are derived from the zeta functions of a Nash function germ introduced in Section 2.2. Recall that the zeta functions are formal power series in the indeterminate $T$ with coefficients in $\mathbb{Z}\left[u, u^{-1}\right]$. The new invariants are obtained from the zeta functions by evaluating $u$ in an appropriate way.

3.1. Evaluating $u$ at -1 . To begin with, let us note that we recover the zeta functions defined by S. Koike and A. Parusiński in [11], which have been proven to be invariants for the blow-analytic equivalence of real analytic function germs, by evaluating the zeta functions of Section 2.2 at $u=-1$.

Indeed, one recovers the Euler characteristic with compact supports by evaluating the virtual Poincaré polynomial at $u=-1$ (cf. Remark 2.7(3)). 
REMARK 3.1. We also recover the zeta functions with sign of [11] of a Nash function germ $f$ as $-2 Z_{f}^{ \pm}(-1, T)$. Indeed, the zeta functions of [11] are defined by considering the value under the Euler characteristic with compact supports $\chi_{c}$ of the set of arcs

$$
Y_{n}^{ \pm}:=\left\{\gamma \in \mathcal{L}_{n}: f \circ \gamma(t)=b t^{n}+\cdots, \pm b>0\right\} .
$$

But the map $\mathcal{X}_{n}^{ \pm} \times \mathbb{R}_{+}^{*} \rightarrow Y_{n}^{ \pm},(\gamma(t), a) \mapsto \gamma(a t)$, is a homeomorphism, therefore

$$
\chi_{c}\left(Y_{n}^{ \pm}\right)=\chi_{c}\left(\mathbb{R}_{+}^{*}\right) \cdot \chi_{c}\left(\mathcal{X}_{n}^{ \pm}\right)=-2 \chi_{c}\left(\mathcal{X}_{n}^{ \pm}\right) .
$$

As a consequence:

Proposition 3.2. Let $f, g:\left(\mathbb{R}^{d}, 0\right) \rightarrow(\mathbb{R}, 0)$ be blow-Nash equivalent germs of Nash functions. Then

$$
Z_{f}(-1, T)=Z_{g}(-1, T)
$$

and

$$
Z_{f}^{+}(-1, T)=Z_{g}^{+}(-1, T), \quad Z_{f}^{-}(-1, T)=Z_{g}^{-}(-1, T) .
$$

\section{REMARK 3.3.}

(1) This is also a direct consequence of the proof of Proposition 2.6 because under a blow-Nash homeomorphism, just the parity of the $\nu_{i}$ is preserved (cf. [11]).

(2) As an application, it follows from [11] that we can give the classification of the Brieskorn polynomials of two variables, $f_{p, q}= \pm x^{p} \pm y^{q}$, $p, q \in \mathbb{N}$, under blow-Nash equivalence, by using the zeta functions evaluated at $u=-1$ and the Fukui invariants. We will see another approach in Section 3.3.

3.2. Evaluating $u$ at 1 . In a similar way, one can evaluate the zeta functions at 1 . In the case of the naive zeta function, what we obtain is only zero! Nevertheless, one can obtain finer invariants. Actually, let us decompose the naive zeta function $Z_{f}(u, T)$ of a Nash function germ $f$ in the following way:

$$
Z_{f}(u, T)=\sum_{l \geq 1}(u-1)^{l} z_{f, l}(u, T)
$$

where $z_{f, l}(u, T)$ is a formal power series in $T$ with coefficients in $\mathbb{Z}\left[u, u^{-1}\right]$ which is not divisible by $u-1$.

Similarly, decompose the zeta functions with sign:

$$
Z_{f}^{ \pm}(u, T)=\sum_{l \geq 0}(u-1)^{l} z_{f, l}^{ \pm}(u, T)
$$

Note that here the summation index may begin at 0 .

By evaluating these series in $\mathbb{Z}\left[u, u^{-1}\right][[T]]$ at $u=1$, one finds new invariants for blow-Nash equivalence. 
Theorem 3.4. Let $f, g:\left(\mathbb{R}^{d}, 0\right) \rightarrow(\mathbb{R}, 0)$ be blow-Nash equivalent germs of Nash functions. Then

$$
z_{f, 1}(1, T)=z_{g, 1}(1, T), \quad z_{f, 0}^{ \pm}(1, T)=z_{g, 0}^{ \pm}(1, T),
$$

and

$$
z_{f, 2}(1, T) \equiv z_{g, 2}(1, T) \bmod 2, \quad z_{f, 1}^{ \pm}(1, T) \equiv z_{g, 1}^{ \pm}(1, T) \bmod 2 .
$$

Note that by mod 2 congruence we mean equality of the series considered as elements in $(\mathbb{Z} / 2 \mathbb{Z})[[T]]$.

REMARK 3.5. If $k \geq 2$, then the series $z_{f, k}^{ \pm}(1, T)$ and $z_{f, k+1}(1, T)$ are also invariant mod 2 , but unfortunately they just vanish!

Proof of Theorem 3.4. This is a consequence of the Denef \& Loeser formula given in Propositions 2.8 and 2.9. Let us concentrate first on the naive case.

Note that

$$
z_{f, 1}(1, T)=\lim _{u \rightarrow 1} \frac{Z_{f}(u, T)}{u-1} \quad \text { and } \quad z_{g, 1}(1, T)=\lim _{u \rightarrow 1} \frac{Z_{g}(u, T)}{u-1},
$$

that is, $z_{f, 1}(1, T)$ (respectively $z_{g, 1}(1, T)$ ) is the derivative with respect to $u$ of $Z_{f}(u, T)$ (respectively $Z_{g}(u, T)$ ) evaluated at $u=1$. One can express these quotients via the Denef \& Loeser formula (Proposition 2.8). As $Z_{f}(u, T)$ and $Z_{g}(u, T)$ are divisible by $u-1$, these quotients coincide up to the coefficients $\nu_{i}$, which only have the same parity. By evaluating $u$ at 1 , we obtain the equality

$$
z_{f, 1}(1, T)=z_{g, 1}(1, T) .
$$

Similarly, $z_{f, 2}(1, T)$ is the derivative of $Z_{f}(u, T) /(u-1)$ evaluated at $u=1$. However, the derivatives of quotients of the type $u^{-\nu} T^{N} /\left(1-u^{-\nu} T^{N}\right)$ appearing in the expression of the Denef \& Loeser formula for $Z_{f}(u, T)$ are of the form

$$
-\nu \frac{u^{-\nu-1} T^{N}}{\left(1-u^{-\nu} T^{N}\right)^{2}}
$$

Therefore the mod 2 congruence of $z_{f, 2}(1, T)$ and $z_{g, 2}(1, T)$ comes from the mod 2 congruence of the different $\nu$.

One just has to repeat the same arguments with $z_{f, 0}^{ \pm}(1, T)$ and $z_{f, 1}^{ \pm}(1, T)$ in order to complete the proof of the theorem in the cases with sign.

EXAMPLE 3.6. Let $f_{p, k}$ be the Brieskorn polynomial defined by

$$
f_{p, k}= \pm\left(x^{p}+y^{k p}+z^{k p}\right), \quad p \text { even, } k \in \mathbb{N} .
$$

It is not known whether two such polynomials are blow-analytically equivalent or not. However, we prove below that for fixed $p$ and different $k$, two such polynomials are not blow-Nash equivalent. 
Note that in [2], we established the analogous result concerning blowNash equivalence via a blow-Nash isomorphism, by using the naive zeta functions. Indeed, the naive zeta function $Z_{f_{p, k}}$ of $f_{p, k}$ looks like

$$
\begin{aligned}
Z_{f_{p, k}}= & (u-1)\left(u^{-1} T^{p}+u^{-2} T^{2 p}+\cdots+u^{-(k-1)} T^{(k-1) p}\right)+\left(u^{3}-1\right) u^{-k-2} T^{k p} \\
& +(u-1)\left(u^{-(k+3)} T^{(k+1) p}+u^{-(k+4)} T^{(k+2) p}+\cdots+u^{-(2 k+1)} T^{(2 k-1) p}\right) \\
& +\left(u^{3}-1\right) u^{-2(k-2)} T^{2 k p}+\cdots .
\end{aligned}
$$

Now, for $p$ fixed and $k<k^{\prime}$, the $p k$-coefficient of $Z_{f_{p, k}}$ is $\left(u^{3}-1\right) u^{-k-2}$ whereas the one of $Z_{f_{p, k^{\prime}}}$ is $(u-1) u^{-k}$. Therefore, the $p k$-coefficient of $z_{f_{p, k}, 1}$ equals 2 whereas the one of $z_{f_{p, k^{\prime}}, 1}$ is 1 , and so $f_{p, k}$ and $f_{p, k^{\prime}}$ are not blowNash equivalent.

3.3. Classification of two-variable Brieskorn polynomials. Effective classification of function germs under a "blow-type" equivalence relation is a difficult topic. In this direction, the simplest example people tried to handle is the one of Brieskorn polynomials. Actually, only the classification of two-variable Brieskorn polynomials has been done completely, under blowanalytic equivalence in [11], and also under blow-Nash equivalence via blowNash isomorphism in [2]. In Remark 3.3, we notice moreover that the invariants used in [11] enable us to get the classification also for blow-Nash equivalence. Here we present an alternative proof using only the invariants derived from the zeta functions.

Recall that two-variable Brieskorn polynomials are of the type

$$
\pm x^{p} \pm y^{q}, \quad p, q \in \mathbb{N} .
$$

As proven in [11], the zeta functions evaluated at $u=-1$ (cf. Remark 3.1) enable one to distinguish the blow-Nash type except in the particular case of

$$
f_{k}(x, y)= \pm\left(x^{k}+y^{k}\right), \quad k \text { even. }
$$

In that case, by the Denef \& Loeser formula we obtain

$$
Z_{f_{k}}(T)=\left(u^{2}-1\right) \frac{T^{k}}{u^{2}-T^{k}},
$$

and if $f_{k}(x, y)=x^{k}+y^{k}$,

$$
Z_{f_{k}}^{+}(T)=(1+u) \frac{T^{k}}{u^{2}-T^{k}}, \quad Z_{f_{k}}^{-}(T)=0
$$

and conversely,

$$
Z_{f_{k}}^{+}(T)=0, \quad Z_{f_{k}}^{-}(T)=(1+u) \frac{T^{k}}{u^{2}-T^{k}}
$$

if $f_{k}(x, y)=-\left(x^{k}+y^{k}\right)$. 
Therefore

$$
z_{f_{k}, 1}=2 \frac{T^{k}}{1+T^{k}}
$$

and thus $z_{f_{k}, 1} \neq z_{f_{k^{\prime}}, 1}$ whenever $k \neq k^{\prime}$, whereas if $k=k^{\prime}$ but the signs are different, the cancellation of $z_{f_{k}, 1}^{+}$or $z_{f_{k}, 1}^{-}$enables one to distinguish $f_{k}$ and $f_{k^{\prime}}$.

As a consequence, we have proved that we can draw the classification under blow-Nash equivalence of the Brieskorn polynomials of two variables, by using the invariants derived from the zeta functions by evaluation of the indeterminate $u$. Moreover, this classification coincides with the ones established in [11] and [2], that is, the blow-analytic type, blow-Nash type via blow-Nash isomorphism and blow-Nash type of the Brieskorn polynomials of two variables are the same.

4. Questions. As we have already noticed, the invariants known for blow-analytic equivalence (the Fukui invariants [10], the zeta functions of S. Koike and A. Parusiński [11]) are invariants for blow-Nash equivalence. However:

Question 4.1. Are the zeta functions $Z_{f}(u, T)$ of a real analytic function germ invariants for blow-analytic equivalence? Or, as a weaker version, are the invariants obtained after evaluation at 1 invariants for blow-analytic equivalence?

More generally, the differences between blow-Nash equivalence and blowanalytic equivalence are not known in the case of Nash function germs or even polynomial germs. As an example, we have proved that the blowanalytic and blow-Nash types of the Brieskorn polynomials of two variables coincide. But in general:

Question 4.2. Does blow-Nash equivalence coincide with blow-Nash equivalence via blow-Nash isomorphism?

QUeSTION 4.3. Does blow-Nash equivalence coincide with blow-analytic equivalence on polynomial germs? On Nash function germs?

\section{References}

[1] J. Denef and F. Loeser, Geometry on arc spaces of algebraic varieties, in: European Congress of Math. (Barcelona, 2000), Vol. 1, 2001, 325-348.

[2] G. Fichou, Motivic invariants of arc-symmetric sets and blow-Nash equivalence, Compos. Math. 141 (2005), 655-688.

[3] -, The corank is invariant under blow-Nash equivalence, Kodai Math. J., to appear. 
[4] T. Fukui, S. Koike and T.-C. Kuo, Blow-analytic equisingularities, properties, problems and progress, in: Real Analytic and Algebraic Singularities, T. Fukuda et al. (eds.), Pitman Res. Notes in Math. Ser. 381, Longman, 1998, 8-29.

[5] T. Fukui, S. Koike and M. Shiota, Modified Nash triviality of a family of zero-sets of real polynomial mappings, Ann. Inst. Fourier (Grenoble) 48 (1998), 1395-1440.

[6] T. Fukui and L. Paunescu, On blow-analytic equivalence, preprint.

[7] T. Fukui and L. Paunescu, Modified analytic trivialization for weighted homogeneous function-germs, J. Math. Soc. Japan 52 (2000), 433-446.

[8] T. Fukui and E. Yoshinaga, The modified analytic trivialization of family of real analytic functions, Invent. Math. 82 (1985), 467-477.

[9] H. Hironaka, Resolution of singularities of an algebraic variety over a field of characteristic zero, Ann. of Math. 79 (1964), 109-326.

[10] S. Izumi, S. Koike and T.-C. Kuo, Computations and stability of the Fukui invariant, Compos. Math. 130 (2002), 49-73.

[11] S. Koike and A. Parusiński, Motivic-type invariants of blow-analytic equivalence, Ann. Inst. Fourier (Grenoble) 53 (2003), 2061-2104.

[12] M. Kontsevich, Lecture at Orsay (December 7, 1995).

[13] T.-C. Kuo, On classification of real singularities, Invent. Math. 82 (1985), 257-262.

[14] K. Kurdyka, Ensembles semi-algébriques symétriques par arcs, Math. Ann. 282 (1988), 445-462.

[15] C. McCrory and A. Parusiński, Virtual Betti numbers of real algebraic varieties, C. R. Acad. Sci. Paris Sér. I 336 (2003), 763-768.

Institut Mathématiques de Rennes

Université de Rennes 1

Campus de Beaulieu

35042 Rennes Cedex, France

E-mail: goulwen.fichou@univ-rennes1.fr

Reçu par la Rédaction le 26.11.2004

Révisé le 11.3.2005 